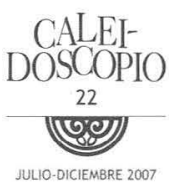

\title{
El problema de la interpretación en las ciencias sociales
}

BENITO LEÓN CORONA

$U A M-I$

D

esde hace mucho, el hombre se ha preocupado y ha generado incontables páginas sobre lo que debe ser entendido como ciencia. Sin la posibilidad de lograr siquiera un recuento mínimo de lo que han sido los temas presentes en esta discusión, es posible identificar uno de las preocupaciones más frecuentes en el desarrollo de la filosofía y la epistemología: ¿el conocimiento sobre la vida social es científico? ¿Puede haber ciencia social? ¿Tiene las mismas características que las ciencias naturales?

Detrás de estas preguntas identificamos la intención de otorgar una explicación de la relación entre el hombre y la naturaleza, y el hombre con el hombre. Para que pueda surgir esta explicación, primero debemos saber cómo se genera el conocimiento, qué procesos ocurren en la mente y en el ser del hombre, pero más aún qué aspectos, más allá de la labor neuronal, ocurren para que el hombre aprenda. Discutir esta problemática, puede ser tarea de la filosofía o de la epistemología, no pretendemos aquí llegar a conclusiones acabadas sobre esta discusión, la intención es modesta sólo pretendemos iniciar el planteamiento de algunas ideas en torno a la formulación de algunos métodos científicos que se han elaborado en los territorios de las ciencias sociales para lograr un acercamiento al problema de la relación hombre-hombre y destacar que no es conveniente hablar de el método científico en singular, pues la pluralidad de perspectivas priva en la actualidad en el quehacer científico. 
Para tal fin, en este ensayo, tratamos de: ordenar algunos textos que hablan de las formas de conocer y aprender en el hombre social, las propuestas metodológicas del positivismo o empirismo lógico y las alternativas propuestas por la fenomenología y la hermenéutica; y ensayar algunas ideas presentes en estas perspectivas sobre el problema metodológico de la Interpretación.

\section{Conocimiento y suieto. El problema de la representación}

Para poder discutir en torno al problema de la Interpretación, es necesario primero dilucidar sobre las características que conforman la construcción del conocimiento en un sentido genérico; para ello, hemos considerado pertinente presentar una breve reflexión al respecto del problema de la representación. Reconociendo que existen variadas alternativas para acercarnos a lo que significa la construcción del conocimiento, el tema de las representaciones ha sido escogido dentro de ellas, ya que ofrece una rica manera de concebir el proceso subjetivo y social en el que se llega a reconocer la construcción de nociones que guían el conocimiento.

Primero, debemos de reconocer que el conocimiento subjetivo lo es en tanto que ocurre en la mente humana a partir de formas racionales en el procedimiento de ordenación y conceptualización que llevan al individuo a formarse una idea de su mundo inmediato. Sin embargo el proceso por el cual el individuo conoce y aprende, no es un proceso aislado, y no supone al sujeto como capaz de crear conocimiento de manera apartada o incomunicada con otros sujetos y con los procesos de los cuales va generándose una imagen que adquiere significado para él.

El proceso es más complejo, en la medida en que el individuo es capaz de observar y verbalizar lo que aprende, se concibe como un ser en relación tanto con la realidad que conoce como con otros sujetos, que hacen de esa realidad algo asible y comprensible para cualquier sujeto social. En corto, se trata de un sujeto social que conoce a partir de las posibles relaciones que establece.

En primera instancia, podemos alejarnos de la idea de aprendizaje generado a partir de la contemplación, proceso que sí correspondería con la idea de un sujeto aislado y pasivo frente a la realidad que conoce. Si hacemos abstracción de esa idea de aislamiento y pasividad del 
sujeto, entonces podremos comprender que la posibilidad de conocer y aprender se realiza como acto social. En la medida en que el sujeto se encuentra inmerso en una variedad de relaciones e interacciones sociales, dentro de un contexto social y cultural o hasta subcultural, el referente del conocimiento se deberá concebir como la "mezcla" de esos elementos "externos" al individuo que le brindan la posibilidad de identificar visual y verbalmente las experiencias de su vida, que le ofrecen información suficiente para operar bajo la lógica de un ordenamiento que le genera conocimiento.

Las representaciones son presentaciones de sus referentes manufacturados activamente, producidas a partir de recursos culturales disponibles!

El problema del conocimiento y el aprendizaje, se vuelven más complejos que la simple contemplación; al tomar en consideración el concepto de representaciones, estamos aludiendo a la condición activa del sujeto en la sociedad. Desde esta perspectiva, entonces definiríamos conocimiento, como ese proceso mental racional, a través del cual el sujeto, construye representaciones a partir de su acción en la sociedad.

Más aún,

cuando una representación proporciona un conocimiento o información sobre, digamos, un objeto, lo hace mediante una clasificación al convertirlo en una instancia de uno o más tipos de entidad reconocida por una cultura, a partir de cuyos recursos se nutre. De este modo, la representación hace posible que el conocimiento existente se aplique a su referente, y convierte al referente en una fuente de información significativa, una contrastación potencial sobre el conocimiento existente. El conocimiento y el objeto están conectados a través de la representación ${ }^{2}$.

Es importante reflexionar sobre lo que hemos señalado con cursivas, ya que si bien podremos estar de acuerdo conque el objeto existe en la realidad sin la acción directa del individuo sobre él, éste no es cog-

Barry Barnes, "El problema del conocimiento", en León Olivé, La explicación social del conocimiento. México, UNAM, 1985, p. 62

Id., p. 60

$\begin{array}{lllllllllllll}C & A & L & E & \text { I } & D & O & S & C & O & P & \text { I } & 0\end{array}$ 
noscible sin que se establezca una relación con el sujeto, y este elabore una significación, y le otorgue sentido. Pero, además, la representación que hace el sujeto del objeto, también se encuentra mediada por otros aspectos, bajo esta perspectiva, no es un conocimiento neutro (abundaremos sobre el asunto más adelante), en cambio, se establece dentro de un contexto determinado cultural y socialmente.

El conocimiento resultado de las representaciones, deberá ser comprendido

como la correlación del desarrollo histórico de los procedimientos, las competencias y técnicas relevantes en grados diversos para los fines y objetivos de culturas y subculturas ${ }^{3}$

Podemos detenernos un momento y reflexionar en torno a cómo se construye el conocimiento científico, si toda representación media la relación entre conocimiento y objeto, este proceso ocurre no sólo en relación con el conocimiento de sentido común que se obtiene a partir de la experiencia cotidiana del "aquí y el ahora", y se le otorga significado a partir de ello, también podremos, en principio, reconocer que el mismo proceso ocurre en la conformación de lo que llamamos conocimiento científico.

Ya la Sociología del conocimiento ha planteado la necesidad de reconocer que los grandes descubrimientos científicos (en ciencias naturales) no sólo son el producto de mentes brillantes y de la virtuosidad (azarosa), de que esos hombres se encontraran en el momento y lugar preciso para lograrlo; se pone en juego el contexto social y cultural en el que se ubican tales avances científicos.

Otro elemento que interesa destacar aquí, está en relación con la neutralidad del conocimiento. Desde la perspectiva que estamos tomando como referente, se hace importante alusión a la obra de Marx, en la que se enfatiza la idea de que el conocimiento está mediado por intereses y actividades; con Marx, se afirma que

3 Ibid. 
los hombres generan conocimiento en el curso de la práctica para preservar sus intereses particulares ${ }^{4}$.

Al establecer que el conocimiento se relaciona con la práctica de los individuos, y reconocer que ésta no es irrestricta; desde la propuesta marxista, se sigue que los intereses de las clases que componen a la sociedad se restringen, y estos a su vez limitan las posibilidades del conocimiento sobre la totalidad de la realidad de los miembros de una clase. Por otro lado, las condiciones históricas de los individuos y de las clases sociales, delimitarán también las posibilidades de generación de representaciones, y con ello de conocimiento.

Podemos concluir esta breve revisión sobre las representaciones haciendo referencia a las formas en que se acumula el conocimiento en las sociedades. A este respecto, el autor nos dice

Si el viejo conocimiento es de hecho una causa material en la generación del nuevo conocimiento, entonces la racionalidad del hombre sola no basta ya para garantizarle acceso a un sólo cuerpo permanente de conocimiento auténtico; lo que puede lograr dependerá de los recursos cognoscitivos que le sean disponibles y de las maneras en que sea capaz de explotar dichos recursos ${ }^{5}$

Esta idea es pertinente en especial para la generación y acumulación de conocimiento en las ciencias sociales, en ellas, se ha afirmado que no es posible hablar de grandes descubrimientos que cambien el rumbo de la ciencia. Más bien, así como en el proceso de conocimiento experiencial del sujeto, se trata de un avance científico que se desarrolla tomando como base las explicaciones e interpretaciones que se han generado a lo largo de la historia. Si esto es así, podemos concluir, que el conocimiento social "acabado" no llegará jamás y que las grandes corrientes del pensamiento científico se originan bajo condiciones peculiares como hoy día que se proponen modalidades teórico-metodológicas para dirigidas a comprender, explicar los procesos de transformación social generados por la globalización.

Id. p 71

Id. $\mathrm{p} 89$

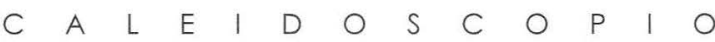




\section{EMPIRISMo LóGico}

En la historia del positivismo podemos encontrar varias etapas, pero interesa destacar aquí la llamada neoclásica o positivismo lógico. Con el Círculo de Viena, esta escuela parte de la finalidad de marcar la frontera entre la metafísica y el conocimiento científico, abogando por una explicación de la realidad, a partir del proceso de verificación, con la aplicación de un método de deducción lógica.

Algunos rasgos metodológicos importantes conforman el núcleo de esta perspectiva:

1. Acuñan la idea de que la ciencia deberá buscar la Ley Universal: entendida como una ley científica que tiene la forma de una proposición universal, pero no es absolutamente verificable. (la noción de Ley Universal, se encuentra en la etapa clásica del positivismo, cuando Comte reconoce que una de las tareas de la ciencia es descubrir tales leyes, que señalan la ocurrencia de "algo" independiente de la voluntad del individuo).

2. Enfatizan en la necesidad de establecer una relación entre pensamiento y realidad a través de la verificación. Se reconoce que ésta no se pueda lograr en algunos enunciados, pero en ese caso, se argumenta que lo que no es verificable es metafísico, y por lo tanto se excluye del objeto de conocimiento de la ciencia positiva.

3. En torno al punto anterior, se acuña la Lógica Simbólica como un lenguaje que crea reglas de razonamiento lógico (equivalente a la matemática). Este lenguaje sería propio de toda ciencia.

4. Para proceder a la verificación, el método remite a la rigurosidad deductiva. De aquí se desprende el método hipotético deductivo, que describiremos más adelante.

5. Las "reglas" para el procedimiento lógico se establecen a partir de la no-intervención de valores, es decir, búsqueda de la objetividad en el establecimiento de la relación entre pensamiento y realidad, que daría como resultado un conocimiento neutro.

6. De aquí se desprende una definición de teoría: "Conjunto de enunciados lógicamente estructurados, semánticamente cerrados, con correlato empirico".

A partir de esta "definición estándar de teoría" y sus implicaciones metodológicas de verificación, se generan posiciones críticas que fundamentalmente cuestionan el papel central que se otorga a la 
verificación y al procedimiento lógico. Destacamos dos críticas, que a su vez produjeron alternativas de análisis:

$1^{\circ}$ Karl Popper propone el proceso de falsación y no de verificación. Este proceso, podría colaborar más en el avance de la ciencia, al reconocer que no todo enunciado lógico puede ser verificado. Vale la pena comentar que la respuesta del neopositivismo a esta crítica de Popper, dice que la ciencia es verificable por convención; es decir, se permite un nivel de adecuación en la ciencia para sostener los postulados básicos de esta teoría.

$2^{\circ}$ Paul Lazarsfeld, sugiere que entre la teoría y la observación empírica deberá establecerse la formulación de hipótesis, para generar indicadores, concebidos como conceptos abstractos y de mayor envergadura, que pueden ordenar y sistematizar al dato empírico. En su propuesta, Lazarsfeld reconoce la inexistencia de un método infalible para construir indicadores, con ello, problematiza un aspecto metodológico que no había sido resuelto por los representantes anteriores del Círculo de Viena: la existencia de un proceso que medie entre el conocimiento lógico y la realidad, a partir de construcciones teóricas.

Si bien Popper y Lazarsfeld introducen matices en el cerrado en tramado del Positivismo Lógico, lo cierto es que el peso de las teorías derivadas de esta forma general de concebir la realidad social, son de gran relevancia en la actualidad, de tal suerte que ha alcanzado el grado de dogma irrefutable, lo que resulta en la reducción de la realidad social al desarrollo de ciertos pasos.

De esta manera el método Hipotético Deductivo derivado del empirismo lógico, vigente hasta nuestros días, señala los "pasos" a seguir para el conocimiento de la realidad social:

$1^{\circ}$ A partir de una teoría, se construyen hipótesis de carácter teórico.

$2^{\circ}$ De una hipótesis teórica se puede construir una hipótesis empírica.

$3^{\circ}$ Se establece el proceso de verificación (o falsación) de las Hipótesis.

$4^{\circ}$ Este procedimiento incluye la delimitación de conceptos e in dicadores que permitan el cumplimiento del paso $3^{\circ}$, pero, más importante, que sirvan como guía de observación. 
La hipótesis, en un sentido general, se define como:

Una pregunta metodológica a la cual el sociólogo intenta responder adecuadamente... es una proposición, condición o principio que es aceptado, para obtener sus consecuencias lógicas, y por medio de un método, comprobar su acuerdo con los hechos conocidos o con aquellos que pueden ser determinados. ${ }^{6}$

O bien

La hipótesis es una expresión conjetural de la relación entre dos o más variables. Siempre aparece en forma de oración aseverativa y relaciona, de manera general o específica, una variable con otra... indica claramente la necesidad de verificar las relaciones expresadas. ${ }^{7}$

Las definiciones anteriores denotan claramente la función que deberá cumplir la hipótesis en el método hipotético deductivo: verificación. Además se refieren a la relación existente entre teoría y práctica, sean hipótesis teóricas, o en un segundo plano empíricas, éstas servirán como guía al planteamiento de procedimientos para el análisis empírico. A partir de ello, podremos establecer la decisión analítica de qué técnicas deberán ser empleadas para el trabajo de investigación empírico.

Es indispensable reconocer que, a partir de los avances experimentados a través de la investigación en este método, el requerimiento de verificación no juega un papel central en los requisitos metodológicos, como se indicaba en los primeros trabajos del empirismo lógico; con ello podríamos decir, se incorpora la posibilidad de falsación, propuesto por Popper. Más aún, en la tarea de verificación, puede ser alterada la hipótesis inicial, en esta perspectiva la hipótesis juega un papel de dirección de la investigación, la importancia ahora radica en ese papel, más que en la propia verificación de nuestras hipótesis iniciales.

Un esquema podría ilustrar las características metodológicas del desarrollo de una investigación bajo esta perspectiva:

6 Antonio Güell, "Hipótesis y variables", en Pierre Boudon y Paul Lazars Feld (comps.), Metodología de las ciencias sociales, Laia, México, 1974, p. 48.

7 Fred Kerlinger, Investigación del comportamiento. Técnicas y metodología, Interamericana, México 1984, p. 12. 

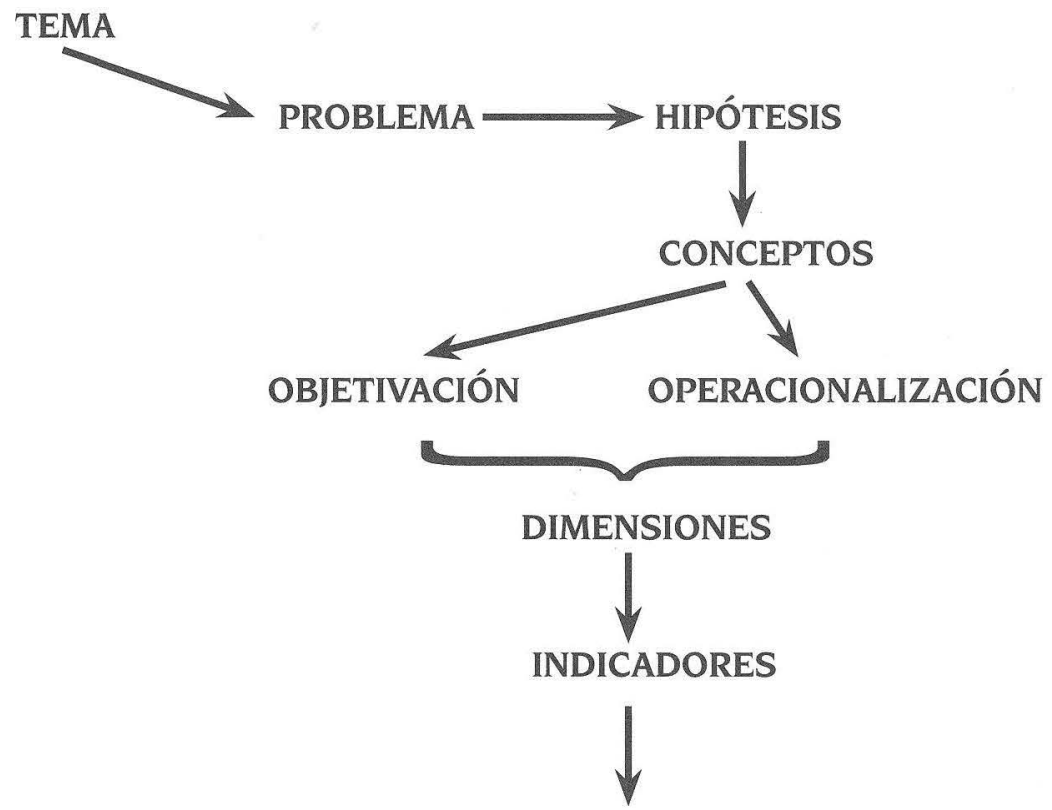

En donde:

TEMA: se refiere a la delimitación de un espacio o fenómeno social general a estudiar.

PROBLEMA: se puede expresar en forma de pregunta, denota una relación entre variables, durante el proceso de investigación se pretende dar respuesta a esa pregunta.

HIPOTESIS: remite al tipo de enunciados lógicos que establecen también una relación entre variables.

CONCEPTOS: considerados como estructuras cognitivas, construidos específicamente para conocer; "denota una abstracción formada por generalización de casos particulares ${ }^{8 "}$ En este nivel, aún nos referimos a conceptos de carácter teórico.

$8 \quad$ F. Kerlinger, op. cit., p. 19

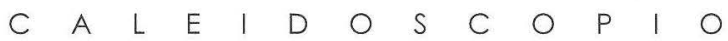


OBIETIVACIÓN: se refiere al recorte empírico para definir qué observar en la realidad.

OPERACIONALIZACIÓN: se refiere al proceso con el que se realiza un recorte lógico de cómo observar a la realidad.

OBJETIVACIÓN Y OPERACIONALIZACIÓN: son los procesos que conducen a la definición de dimensiones e indicadores.

DIMENSIONES: refiere a la generación de conceptos más específicos, dimensionalizar los conceptos se refiere al proceso de conferirle un contenido más concreto.

por regla general, la complejidad de los conceptos utilizados en Sociología es tal que su traducción operativa exige una pluralidad de dimensiones. ?

INDICADORES: refiere a un nivel aún más concreto que la dimensionalización de los conceptos teóricos, dan contenido a las dimensiones. "representan propiedades observables de las unidades (objetos) en estudio ${ }^{10 "}$. Como parte del proceso de operacionalización se conectan los conceptos observables (dimensiones) con los indicadores. Se afirma que este es el último enlace entre teoría y realidad.

Hasta aquí hemos intentado una descripción básica sobre el contenido y propuestas del método hipotético deductivo, y de los procedimientos metodológicos que señala para la realización de un trabajo de investigación. Antes de presentar algunas técnicas de investigación derivadas de esta propuesta metodológica, expondremos otras vertientes de análisis alternativa, que implican otros métodos de investigación.

9 P. Lazarsfeld, "De los conceptos a los índices empíricos", en Boudon y Lazarsfeld op. cit., p. 38.

10 Fernando Cortés y Rosa María Ruvalcaba, Escalas básicas de medición, México, FLASCO, 1985, (mimeo). 


\section{La Fenomenología y la Hermenéutica}

\section{a. La fenomenología}

Esta perspectiva de análisis en las ciencias sociales y en particular en la sociología, nace con la propuesta de Edmund Husserl de analizar los fenómenos sociales a partir de la experiencia subjetiva de los individuos; el individuo se constituye como el objeto de estudio, a partir de tres elementos: Primero, la definición del "yo" como sujeto que experimenta, que vive, percibe y significa los fenómenos sociales que le rodean;

En segundo lugar, hay actos de vida consciente: está el recordar, el descubrir, el conocer, el temer, el imaginar, el gustar. En tercer lugar, existen objetos de consciencia: citas que se recuerdan, gente a la cual se teme, comidas que se apetecen. En resumen, el dominio fenomenológico tiene una estructura tripartita que consiste en yo, cogitaciones y cogitatum. El hecho de que la conciencia sea siempre consciencia de algo, fue llamado por Husserl la estructura intencional de la conciencia'l.

El estudio de los fenómenos sociales deberá realizarse a partir de la concepción de que el fenómeno es y existe "tal y como" ha sido experimentado por el sujeto. En este sentido las percepciones y símbolos así como los significados que se adquieren con la experiencia constituyen el objeto de estudio de la fenomenología. La forma de representación de esas percepciones, símbolos y significados del sujeto, son los "actos internos y los actos mentados según son mentados", a esto llamaríamos actos de conciencia.

Al generar un procedimiento para el estudio de los fenómenos sociales, tal cómo ocurren para el sujeto, la fenomenología no se plantea el problema de la objetividad,

La fenomenología ha sido utilizada para sensibilizarnos respecto de la importancia del sujeto (como analista) como fuente de todo conocimiento ${ }^{12}$.

11 H. Schwartz y J. Jacobs, Sociología cualitativa, México, Trillas, 1996, p. 448.

12 Id. p. 453.
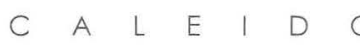

S

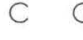


De alguna manera, estas ideas generadas por la fenomenología, nos llevan a reflexionar sobre lo expuesto en la primera parte del ensayo: las representaciones, como mecanismos en los que se manifiesta la forma en la que procede el sujeto para generar conocimiento; como lo habíamos mencionado, la generación de conocimiento y aprendizaje del sujeto, se encuentra contextualizado por la cultura, por la historia, por la posición que se tiene en la sociedad, en general por la experiencia, aspecto central en el análisis fenomenológico.

\section{La Hermenéutica}

Una tercera perspectiva, de alguna manera emparentada con la fenomenología, es la hermenéutica, que puede ser definida, sin ahondar en ello, como la explicación o interpretación del pensamiento que nace a partir del trabajo de interpretación de las sagradas escrituras. Se reconoce a Diltey como una de sus principales exponentes, quien afirma que no se trata sólo de una técnica, y se aleja de la arbitrariedad interpretativa y del naturalismo. Para proceder con este método debe existir un previo conocimiento de los datos históricos o filosóficos de la realidad que se trata de comprender, pero da sentido a los datos por medio del proceso circular de la comprensión. Pasa de los signos a las vivencias, pero se concibe como una ciencia de mayor alcance que la psicología. Heidegger, por su parte, afirma que se trata de un "método de pensar originariamente", (más allá de la fenomenología Husserliana).

Con la hermenéutica, se desarrollan y consolidan una serie de estudios de carácter sociológico y antropológico, que se ubican en el análisis de las formas y manifestaciones culturales. A este respecto, vale la pena recuperar el trabajo de John B. Thompson quien sugiere la elaboración de un marco metodológico general en el que puede ser situada la descripción densa: la Hermenéutica profunda, que pone de relieve el hecho de que el objeto de análisis es una construcción simbólica significativa que requiere de una interpretación.

Thompson destaca que el contexto es muy importante, el trabajo de interpretación no debe descuidar los espacios sociales de la vida cotidiana, el campo de estudio se encuentra compuesto por una 
serie de formas simbólicas que son preinterpretadas por los sujetos, según su contexto. Vale la pena destacar, la propuesta de Thompson al respecto de los componentes o fases del proceso interpretativo de la hermenéutica profunda:

1) Análisis sociohistórico. Las formas simbólicas no se encuentran en el vacío, sino que se transmiten y reproducen en condiciones sociales e históricas específicas. Con ello propone desarrollar un proceso en el estudio que lleve a desentrañar las características de este contexto social e histórico, al incorporar elementos como los distintos escenarios en los que ocurren las formas simbólicas; las Instituciones juegan un papel importante, ya que constituyen los espacios que permiten reproducir las reglas, y mecanismos de control sociales. Aquí se encuentran también los estudios referidos a la estructura social, y los medios técnicos de transmisión, (para este autor ha sido de gran importancia estudiar la comunicación masiva).

2) Análisis formal o discursivo. Aquí se incorporan algunas técnicas y métodos de estudio propiamente lingüísticos, como la semiótica, el análisis conversacional, el sintáctico, narrativo, y argumentativo. En la medida en que el lenguaje representa la forma más acabada de comunicación, es importante para Thompson reconocer la importancia de la construcción de significados en el uso del lenguaje y del discurso social.

3) Interpretación/reinterpretación, con lo que se refiere al proceso de análisis que otorga un significado a aquellas formas culturales que han adquirido un significado previo en las acciones sociales. Por ello es que llama a este proceso de reinterpretación al reconocer los aspectos constitutivos del significado en las acciones y discursos sociales, es importante en este proceso la vinculación con el análisis de discurso. Destaca aquí el "aspecto referencial" de las acciones. Estos tres procesos, no son fases que se desarrollen como un continuo, sino que se realizan en paralelo, tratando de desentrañar la explicación e interpretación de los distintos significados en contextos específicos.

Con estas propuestas, alternativas al método hermenéutico profundo, es posible reflexionar en torno a cómo se construye en

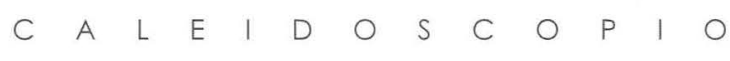


conocimiento en las ciencias sociales, ya podemos alejarnos de la propuesta inicial de que el conocimiento científico se produce de la misma manera que el conocimiento del sentido común, no se trata sólo de representaciones, se elabora a partir de procedimientos y métodos que, con determinados énfasis en el cómo, ordenan la "información" procedente de la realidad, buscando su comprensión y explicación. Son, en su caso, representaciones de "mayor envergadura" y que tienen detrás una cuestión central para el conocimiento: las teorías. Es decir, conocimiento acumulado.

\section{LAS TÉCNICAS EN LOS MÉTODOS}

Aunque la distinción entre métodos cualitativos y cuantitativos como métodos, pueden ser relacionados directamente con las propuestas metodológicas empirista y fenomenológica o hermenéutica respectivamente, el quehacer científico no se limita a estas relaciones. Es decir, a partir del método hipotético deductivo, en algún momento del análisis (a partir de la generación de hipótesis) se puede optar por el uso de algunas técnicas de orden cualitativo, que generalmente se identifican con la otra perspectiva. Con ello, queremos decir que las técnicas de investigación no constituyen una forma de propiedad de los métodos en que se han desarrollado. Sin embargo, para facilitar la descripción, hablaremos de las técnicas, a partir de los métodos que las han propuesto.

Brevemente, podemos decir que del método hipotético deductivo, se desprende algunas técnicas para el análisis de fenómenos sociales, destacamos la encuesta y el censo. Estas técnicas, representan lo que se ha llamado análisis cuantitativos, en la medida en que sostienen sus procedimientos en la cuantificación de características de los individuos para explicar un fenómeno social. Dentro de estos métodos cuantitativos, la encuesta, parte de la delimitación de una muestra estadística, de la población a estudiar. Busca producir información generalizable y adquiere una representación numérica (geométrica y matemática). El censo, por su parte, abarca a una población total, y persigue una descripción exhaustiva de la población.

Por su parte, las propuestas metodológicas de la fenomenología y la hermenéutica, han generado técnicas de carácter cualitativo, en 
franco alejamiento de las técnicas antes descritas. Buscan encontrar información específica, no cuantificable, y no adquieren una representación numérica. Entre otras, destacan la Historia de Vida, la Entrevista a profundidad, el Análisis de Discurso; en estas técnicas se busca profundizar en la comprensión de la acción de los sujetos y sus significados y como ya hemos dicho, no se cuestionan sobre el problema de la objetividad.

\section{EL PROBLEMA DE LA INTERPRETACIÓN}

Antes de iniciar propiamente con la discusión al respecto de la interpretación en los métodos y técnicas de investigación antes descritos, es necesario reconocer que la idea de interpretación, desde luego, surge de las propuestas metodológicas fenomenológicas, y más precisamente hermenéuticas (de donde más explícitamente se ha tomado el método). Con ello, es importante también reconocer que este tipo de métodos han sido desarrollados en estudios sobre la cultura, y en particular a partir de la Antropología en su especialización como disciplina de la cultura. De esta manera, los referentes para llegar a delimitaciones más precisas provienen de este método, esta disciplina y este campo de análisis. Por otra parte, conviene aclarar que para la discusión sobre la interpretación echaremos mano de ejemplos diversos para ilustrar las reflexiones.

A partir de lo anterior, en primer lugar, es necesario establecer algunas consideraciones como punto de partida: la realidad social, en la medida en que se encuentra integrada e influida por aspectos culturales, está permeada por la construcción de formas simbólicas:

Las formas simbólicas son construcciones significativas que requieren una interpretación; son acciones, expresiones y textos que se pueden comprender en tanto que construcciones significativas. Ese énfasis fundamental en el proceso de comprensión e interpretación mantiene su valor hoy ${ }^{13}$.

En este terreno Heidegger ha propuesto que el proceso de comprensión, relacionado con la construcción significativa, no opera de

13 John B. Thompson, Ideología y cultura moderna. Teoría crítica social en la era de la comunicación de masas, México, UAM-X, 1990, p. 301. 
manera distinta en la comprensión científica y en la vida cotidiana, "comprender es algo que nosotros, en tanto seres humanos, hacemos todo el tiempo de todas maneras, y los procedimientos de interpretación más especializados que emplean los analistas sociales dan por sentadas las bases preestablecidas de la comprensión cotidiana, y se inspiran en ellas" (Thompson: 302) De esta forma la vida cotidiana genera, igualmente, interpretaciones de la realidad social las que el experto retoma para trabajar de manera sistemática, a través de instrumentos conceptúales, y así lograr construcciones que permitan comprender y explicar la realidad social de forma científica.

Con ello, se afirma que el proceso de comprensión del analista social, se elabora sobre la base de las interpretaciones que hacen los sujetos en ese mundo de símbolos y significados, es entonces una proceso de "reinterpretación de la interpretación". ${ }^{15}$

Por su parte, Medina Echavarría, afirma que

Los datos de las ciencias sociales y culturales están penetrados por símbolos; lo mismo los objetos que las conductas. Símbolos que pueden ser conocidos con todo rigor ya interpretando lo que la gente dice, declara o comunica respecto de ellos; bien observando la conducta externa de esa gente en relación con determinados objetos. ${ }^{16}$

¿Qué y cómo son esos datos? Estos pueden ser "objetivos" en la medida en que refieren a características "medibles", cuantificables, del comportamiento social (no debemos olvidar que se refleja en símbolos y adquieren significado). Si tomamos como ejemplo el análisis del trabajo académico, podemos operar a partir de técnicas cuantitativas para conocer, por ejemplo, cuál es el tipo de contrato que tienen los académicos en la Institución en la que trabajan. Se trata de un dato

15 A partir de ello, se estructura la tercera fase de la hermenéutica profunda de Thompson, descrita con anterioridad. Esta cobra significado a partir de la idea de la comprensión de los fenómenos sociales, preinterpretados por los sujetos.

16 José Medina Echavarría, "Concepto y realidad", en Sociología, teoría y práctica, México, FCE, 1987, p. 100. 
objetivo, medible y "agrupable" en variables que nos permitan distinguir grupos de académicos en diversas formas de contrato. Pero, aquí entra la vieja frase casi coloquial de que "los datos no hablan por sí solos"; una vez que hemos agrupado a los individuos, podemos observar sus características contractuales, pero nuestra intención es explicar esas formas de contratación; aquí debemos incorporar nuestra experiencia, nuestra representación de lo que significa estar contratado de una manera u otra, implica reinterpretar el significado que socialmente se otorga a la posición contractual de un trabajador.

Pensando en un ejemplo más simple, si queremos caracterizar a los grupos de académicos por genero, una primera fase del proceso es conocer cuantos son hombres y cuantas mujeres, pero en el momento de intentar una explicación al respecto, nuevamente se pone en juego un proceso de interpretación de lo que significa ser académica o académico. Con estos ejemplos, lo que queremos ilustrar es que, si procedemos a partir de un método hipotético deductivo, a estas alturas no podemos quedarnos con la simple caracterización y descripción de los datos. Si procedemos a la verificación de las hipótesis planteadas al inicio, debcmos interpretar los datos, por más objetivos que estos sean.

Otro tipo de datos refieren a las actitudes, los valores y las percepciones, estos datos evidentemente tienen un correlato interpretativo y reinterpretativo más claro. De cualquier forma podemos proceder a través de técnicas cuantitativas para obtener los datos, podemos hacerlo a través de escalas: Likert, o de Diferencial Semántico. Los resultados son cuantificables, pero aquí más que en otro tipo de variables, los resultados deberán ser interpretados, tomando en cuenta el contexto cultural, social, político (y hasta ético) de los individuos que estudiamos.

Es claro que no se trata de una interpretación de las variables identificables a un sujeto, en la medida de que si tomamos como referente una muestra, se busca con ello llegar a generalizaciones, el proceso de interpretación, deberá contemplar aspectos culturales y sociales también generalizables.

Por otro lado, los métodos cualitativos, basados en la fenomenología propone Lines, "pretenden superar la dicotomía" y el "mundo

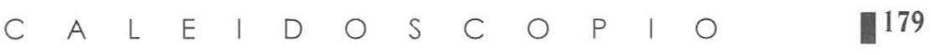


externo", integrándolo en la percepción del sujeto (...) el objeto o mundo externo es subsumido en el sujeto..., cómo establecer la relación entre el nivel de lo individual con el de lo social. Este problema puede encontrar respuesta en la medida en que dicha relación sea concebida como una nueva síntesis..., en la medida en que ambos niveles se conciban como parte de un totalidad que es cambiante"17

El problema de la interpretación, en los métodos cualitativos, se relaciona con ese proceso de integración, ya que para exponer, por ejemplo, una historia de vida, el sujeto, que es quien relata ya está otorgando sentido e interpreta sus acciones de alguna manera; por otro lado, el investigador tratará de exponer su interpretación sin mezclarla o contaminar con ella las interpretaciones del mismo sujeto. Es propiamente un proceso de reinterpretación.

Ahora bien, huelga enfatizar que no se trata de esquemas de in terpretación "libres" de lo que cree el investigador que debe ser. Los métodos cualitativos, y la hermenéutica se han ocupado de insistir en que estos procesos de interpretación deberán ser llevados a cabo a partir de un procedimiento riguroso de relación entre los sujetos y el contexto histórico-social en que se desarrollan las acciones de los sujetos, este aspecto, permitirá "verificar" si el proceso de interpretación tiene validez en el análisis.

Esto nos lleva a destacar que en todo proceso de investigación social, es necesario tener presente siempre la relación entre sujeto y sociedad, historia y momento actual, en las acciones de los sujetos, inmersos en fenómenos sociales.

\section{Comentario Final}

Queremos cerrar este trabajo, abriendo al mismo tiempo las puertas de la investigación a través de las siguientes consideraciones: "la vida del hombre se despliega en campos cada vez más amplios y ricos en nuevas esperanzas, lo que contribuye a que el "ser" del hombre con -

Montserrat Lines (et al.), La historia de vida, México, UNAM-Porrúa, 1988, p. 92. 
lleve una constante ampliación de sus horizontes de vida, pues lo que ayer no era valorado, e incluso se desconocía, más tarde se convierte en una exigencia de primera importancia.

Esta transformación que experimenta el contenido del mundo real como vivencia de privativo que cada uno posee y "lo que le es externo pero que representa la realidad que puede conquistar", establece Hugo Zemelman.

O bien, al reconocer que las posibilidades de autorrealización, en los términos expuestos antes, encuentran sus limitaciones en una realidad cada vez más agreste, marcada por la perdida de alternativas para erigirse en el forjador del destino propio, De hecho la labor actual del analista social es comprender y explicar la complejidad de la realidad social y lograr interpretaciones adecuadas a los aspectos que sean de su interés. 
